\title{
Electron Spin Resonance of Gamma Irradiated Single Crystals of Methyl Glycinate Hydrochloride
}

\author{
Mehmet Birey \\ Physics Department, Faculty of Sciences, Ankara University, 06100 Tandoğan Ankara, Turkey \\ Reprint requests to Dr. M. B.; E-mail: birey@science.ankara.edu.tr
}

Z. Naturforsch. 57 a, 36-38 (2002); received December 10, 2001

Two radicals, formed from methylglycinatehydrochloride, $\mathrm{C}_{3} \mathrm{H}_{8} \mathrm{ClNO}_{2}$, crystals on irradiation with $\gamma$ rays, have been identified as $\mathrm{CH}_{2} \dot{\mathrm{C}} \overline{\mathrm{OOCH}}{ }_{3}$ and $\dot{\mathrm{C}} \overline{\mathrm{O} O C H} \mathrm{H}_{3}$.

The electron spin resonance ESR technique has frequently been used for identifying irradiation damage centres in organic and inorganic molecular samples [1]. The ESR spectra of oriented free radicals in many organic single crystals have now been measured $[2,3]$. The observed species are usually produced by gamma irradiation and measured at room temperature. The ESR spectra of $\gamma$-irradiated single crystals of $\mathrm{C}_{3} \mathrm{H}_{8} \mathrm{ClNO}_{2}$ (MGHC) have been observed between $110-300 \mathrm{~K}$ in case of $H \| x$ by recording them. The single crystal spectra were taken at 5 degree intervals for the magnetic field $\mathrm{H}$ being applied in each of the three crystallographic planes, $a b, b c$, and $c a$ and indicated approximately the $1: 5: 10: 10: 5: 1$ and 1 : $3: 3: 1$ intensity pattern shown in Fig 1 , (a) and (b), respectively.

We have attributed them to the $\mathrm{CH}_{2} \dot{\mathrm{C}} \overline{\mathrm{OOCH}}{ }_{3}$ and $\dot{\mathrm{C} O} \mathrm{OCH}_{3}$ radicals. The spectra were found to be temperature dependent between 110 and $300 \mathrm{~K}$. The set of lines in Fig. 1(a) appeared to be unchanged, except for a small anisotropy in the line splitting, and the $g$ value was taken in all spectra. Therefore, the radical responsible for this spectrum must contain five protons in the $\mathrm{CH}_{2} \dot{\mathrm{CO}} \overline{\mathrm{OCH}} \mathrm{H}_{3}$ radical rotating about a symmetry axis at room temperature for all orientations of the magnetic field. This spectrum consists of 6-lines caused by the $\mathrm{CH}_{2} \dot{\mathrm{C}} \overline{\mathrm{OOCH}} \mathrm{CH}_{3}$ radical. The radical splits into $1: 3: 3: 1$ lines because of the protons of the $\dot{\mathrm{COOOCH}} 3$ radical, then each line also splits into $1: 2: 1: 3: 6: 3: 3: 6: 3: 1: 2: 1$ lines due to the protons of the $\mathrm{CH}_{2} \dot{\mathrm{CO} O}$ radical. No splitting due to the NH could be observed. The ESR spectrum for a larger number of inequivalent protons

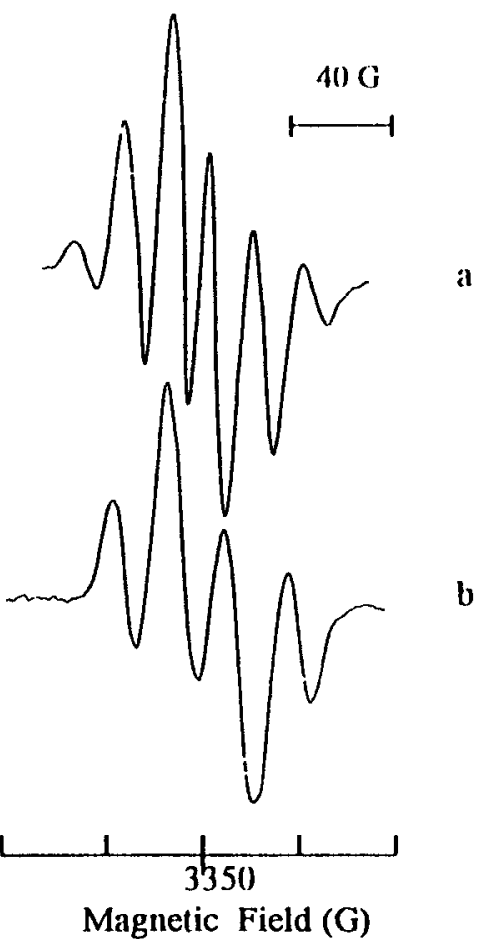

Fig. 1. ESR spectra of $\gamma$-irradiated MGHC a) at $300 \mathrm{~K}$, b) at $110 \mathrm{~K}$.

can be found by graphic construction or by the binomial expansion which is a mathematical record of an ESR spectrum. Straight forward addition of the line intensities of simple spectra, the lines being displaced relative to each other by the value of the coupling constant, yields the intensity ratio for more complicated spectra. 


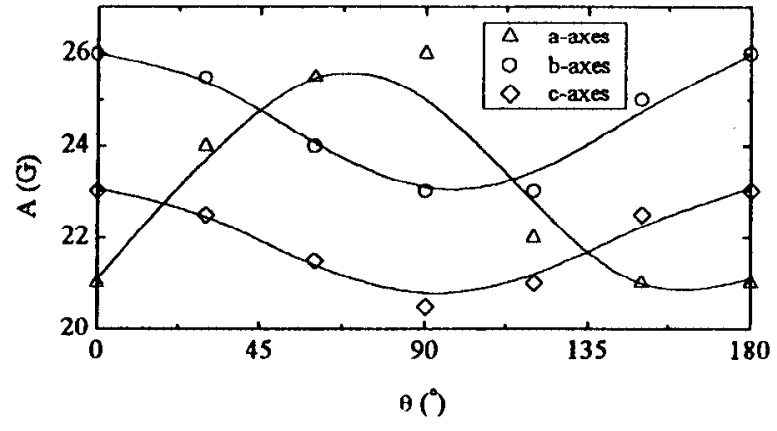

Fig. 2. The angular variations of the A-tensor for the $\dot{\mathrm{CO}} \mathrm{OCH}_{3}$ radical.

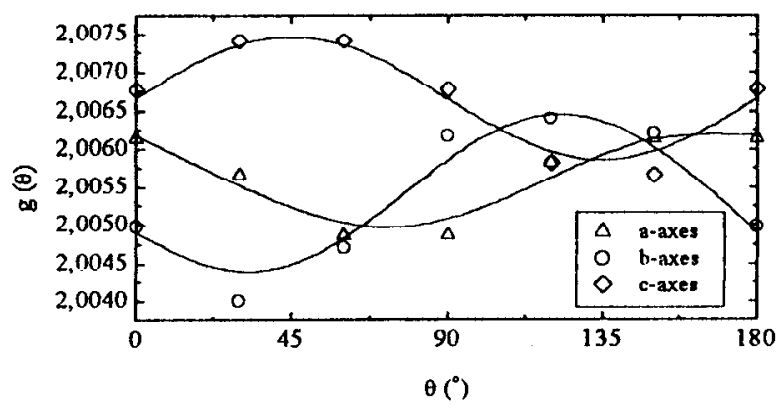

Fig. 3. The angular variations of the g-tensor for the $\dot{\mathrm{CO}} \mathrm{OCH}_{3}$ radical.

The hyperfine splitting of the methyl protons is isotropic, and the hyperfine constant $\mathrm{a}$ is $19 \mathrm{G}$. The $g$ value of this radical is slightly anisotropic, its average value being $g=2.0048$ for the $\dot{\mathrm{C}} \overline{\mathrm{OOCH}}{ }_{3}$. The hyperfine interaction of the protons in the $\mathrm{CH}_{2} \dot{\mathrm{CO} O}$ radical with the unpaired electron is isotropic, its value being a $\left(\mathrm{CH}_{2} \dot{\mathrm{CO} O}\right)=19 \mathrm{G}$. These ESR spectra obtained from the second radicals at $110 \mathrm{~K}$ were not identical with those taken at room temperature. A preliminary analysis indicates that the intensity ratio shown in Fig. 1(b) is due to three magnetically equivalent and nearly isotropic protons. This strongly suggests that the second radical created in gamma irradiated MGHC is the $\dot{\mathrm{CO} O C H}{ }_{3}$ radical. The isotropic component of the hyperfine interaction tensor is $1 / 3(\operatorname{Tr} A)=$ $18 \mathrm{G}$ for the $\mathrm{C}^{\circ} \mathrm{OCH}_{3}$. This value agrees also with alpha protons coupling for methyl radical [4 - 7]. The variations of $A(\theta)$ and $g(\theta)$ are given in Fig. 2 and Fig. 3, respectively. The principal values with thcir direction cosines are given Table 1.

At the low-temperature, the hyperfine interaction of the protons in the MGHC fragment with the unpaired electron was previously attributed to the interaction of
Table 1. The ESR parameters of the radical $\dot{\mathrm{CO} O C \mathrm{OH}_{3}}$ observed in $\mathrm{C}_{3} \mathrm{H}_{8} \mathrm{ClNO}_{2}$ at $110 \mathrm{~K}$.

\begin{tabular}{lcrrr}
\hline \multicolumn{4}{l}{ Principal values } & \multicolumn{4}{l}{ Direction cosines } \\
\hline$a$ & 26,39 & 0,935 & 0,131 & 0,329 \\
& 22,89 & $-0,128$ & 0,991 & $-0,046$ \\
& 20,34 & $-0,331$ & 0,003 & 0,943 \\
$a_{\text {av }}$ & 23,21 & & & \\
$g$ & 2,0093 & 0,305 & $-0,605$ & 0,735 \\
& 2,0057 & 0,949 & 0,254 & $-0,184$ \\
& 2,0019 & $-0,075$ & 0,754 & 0,652 \\
$g_{\text {av }}$ & 2,0056 & & & \\
\hline
\end{tabular}

the spin on the $\mathrm{CH}_{2} \dot{\mathrm{C}} \overline{\mathrm{OOCH}} \mathrm{C}_{3}$ group with the $\mathrm{H}$ protons. The magnitude of these splittings depends on the dihedral angle $\alpha$ between the plane passing through the $\mathrm{O}-\mathrm{C}$ bound and the $\mathrm{p}$ orbital which contains the unpaired electrons. The splitting is given as

$$
\alpha_{\mathrm{H}}=H_{0}+H_{1} \cos ^{2} \alpha
$$

where $H_{0}$ is a constant, and includes the contributions from the spin density which arises from the conformation independent mechanisms, in particular the spin polarisation, and $H_{1}$ includes the hyperconjugative contributions. In the case of rapid rotation about the $\mathrm{OCH}_{3}$ bond, the average value of $a_{\beta}$ becomes

$$
a_{\beta}=H_{0}+\frac{1}{2} H_{1} \text {. }
$$

The constants $H_{0}$ and $H_{1}$ have been experimentally determined as $3,5 \mathrm{G}[8,9]$ and $32,1 \mathrm{G}[10]$, respectively. If these values are replaced (1) and (2), $a_{\mathrm{H}}=$ $16,05-19,55 \mathrm{G}$ and $a_{\beta}=18-19 \mathrm{G}$ is obtained in this study.

The spin densities at the oxygen atoms bonded to carbon can be evaluated from experimental proton splittings by using Mc Connell's equation [10] $a_{\mathrm{H}}=$ $\rho Q ; \rho=0,664$.

\section{Experimental}

MGHC single crystals were grown by slow evaporation of aqueous ethanol solutions. The crystals were irradiated at room temperature by ${ }^{60} \mathrm{Co}-\gamma$ ray source of $0.3 \mathrm{M} \mathrm{radh}^{-1}$ for 24 hours. The spectra were recorded with a Varian E109 C model ESR spectrometer using $2 \mathrm{~mW}$ microwave power. The crystals were rotated on a Lucite pillar about their crystallographic axes. The experiments were carried out many times with several single crystals. The $g$ factor was found by comparing with a DPPH sample $(g=2.0036)$. 
[1] N. M. Atherton, Electron Spin Resonance, J. Wiley, NewYork 1973, p. 103. (1973).

[2] F. Köksal, M. Birey, R. Tapramaz, and F. Çelik J. Mol. Struct. 221, 283 (1990).

[3] M. Birey, H. Aktaş, A. Alicilar, and M. Gürü, Radiat. Eff. and Defects in Solids 140, 365 (1997).

[4] I. Miyagava and W. Gordy, J. Amer. Chem. Soc. 83, 1063 (1961).

[5] M. T. Rogers and L. D. Kispert, J. Chem. Phys. 461, 221 (1966).
[6] J. R. Morton, Chem. Rev. 64, 453 (1964).

[7] J. E. Bennet and L. H. Gale, Trans. Faraday Soc. 64, 1174 (1968).

[8] R. W. Fessenden and R. H. Schuler, J. Chem. Phys. 39, 2147 (1968).

[9] W. T. Dixon, R. O. C. Norman, and A. L. Bulay, J. Chem. Soc. 3625 (1964).

[10] M. C. R Symons, J. Chem. Soc., Part 1, 277 (1959). 